ISSN 1112-9867

http://www.jfas.info

\title{
THE KNOWLEDGES OF TRADITIONAL IRRIGATION IN THE OASIS OF KERZAZ IN SOUTHWEST ALGERIAN: LEGACY AND DEVELOPMENT
}

\author{
C. $\operatorname{Rezzoug}^{1}$, B. Remini $^{2}$ and S. Hamoudi ${ }^{1}$ \\ ${ }^{1}$ Faculté de génie civil et d'architecture, université de Chlef, Hay Salem, route nationale $\mathrm{N}^{\circ}$ \\ 19, 02000 Chlef, Algérie. \\ ${ }^{2}$ Faculté de technologie, université de Blida, route de Soumâa BP 270 \\ 09000 Blida, Algérie.
}

Received: 28 May 2016 / Accepted: 27 December 2016 / Published online: 01 January 2017

\begin{abstract}
In the valley of Saoura, the demand for water for irrigation has increased significantly and rapidly, it requires mobilization and rational and intensive use of all existing water sources like the groundwater which are the only source of drinking water supply and irrigation in the region and the protection of the large vein that feeds the Saoura and bearing his name "oued Saoura".

Oasis Kerzaz is amongst the the most celebrated oasis and the biggest of valley of Saoura, it now suffers from several impediments to their development as: the scarcity of irrigation water, land abandonment, the silting up, the chunking and the exiguity of agricultural land, the food nature of agricultural activity and incurable diseases of crops.
\end{abstract}

Keywords: palm grove; well; Chadouf; Khottara; motorpump; Saoura.

Author Correspondence, e-mail: cherifrezzoug@yagoo.fr

doi: http://dx.doi.org/10.4314/jfas.v9i1.17 


\section{INTRODUCTION}

L'agriculture à Kerzaz est du type oasien, elle est alimentée en eau par une seule façon, les irrigants de Kerzaz exploitent les eaux souterraines, ces eaux représentent l'unique source d'approvisionnement en eau d'irrigation et en eau de boire pour les habitants de Kerzaz. Le droit d'eau était généralement propriété privée par l'exploitation des puits à balancier (Khottara ou bien puits à chadouf) et des forages [1]. Le système traditionnel connaisse des problèmes croissants et finissent par disparaître de la majorité de ces puits à chadouf.

Malgré la rudesse du climat, l'insuffisante de la pluviométrie et le milieu hostile, les oasiens grâce à leur intelligence utilisaient une technique d'irrigation géniale (puits à chadouf) pour cultiver ses terres sans perturber un écosystème fragile. Mais l'envahissement des systèmes modernes d'irrigation comme les motopompes a complètement détruit un environnement propre.

L'objectif de notre travail est d'étudier l'impact de l'invasion des systèmes modernes d'irrigation (réalisation du barrage de Djorf Torba, la propagation anarchique et irrationnelle des motopompes) sur l'utilisation des techniques ancestrales de captage et de partage des eaux dans l'oasis de Kerzaz, le rabattement des nappes de l'oasis et la salinité des eaux et des sols de l'oasis.

\section{MATERIEL ET METHODES}

\subsection{Présentation de l'oasis}

Kerzaz à un patrimoine culturel ayant Enraciné dans les profondeurs de l'histoire, elle est située au Sud-ouest algérien, à $330 \mathrm{~km}$ au sud de Béchar et à $250 \mathrm{~km}$ d'Adrar (figure 1) [2]. C'est une daïra qui englobe les trois communes:

- Kerzaz

- Beni Ikhlef

- Timoudi 

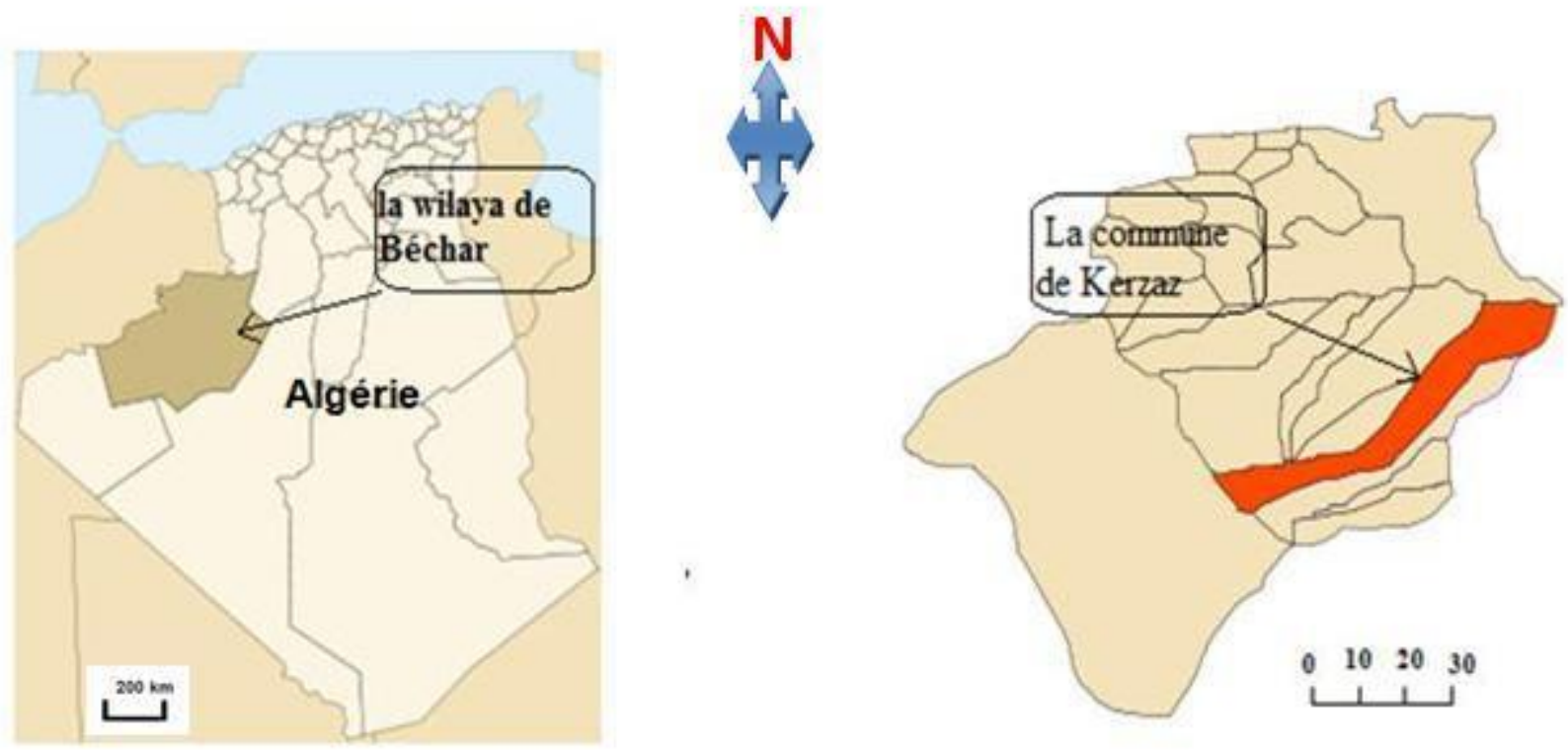

Fig.1. Localisation de la commune de Kerzaz [2].

Le ksar de Sidi Moussa (le grand Cheikh de Kerzaz), au Sud de la zaouia El-Kbira (figure 2), est considéré comme étant le noyau le plus ancien de Kerzaz. Il date de la période du VIIIème au IXème siècle de l'hégire.

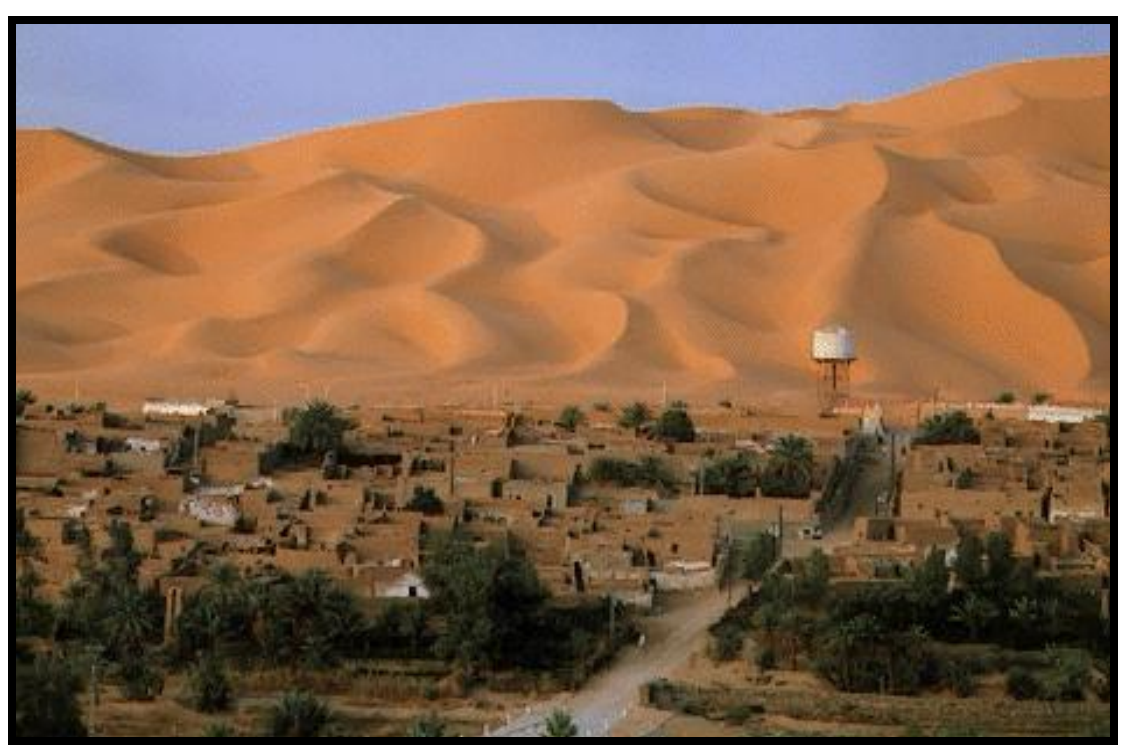

Fig.2. Le vieux ksar de Kerzaz. 2012

La population totale de la daïra de Kerzaz est de 10076 habitants pour une superficie de $19310 \mathrm{~km}^{2}$. La population active représente $27.94 \%$ de la population totale de Kerzaz et $13.6 \%$ de la population active travaille en agriculture [2]. L'agriculture pratiquée dans cette oasis est généralement du type intensif sous les palmiers dattiers. Kerzaz est considérée 
comme un espace oasien avec toutes ses composantes naturelles, socioculturelles et socio-économiques. Le passage technologique d'une agriculture traditionnelle à une agriculture moderne bouleverse profondément l'état des sources non renouvelables comme les nappes souterraines, la production journalière d'eau destinée à l'agriculture s'élève en 2013 à $3649,60 \mathrm{M}^{3}[2]$.

\subsection{Le climat de l'oasis}

Oasis de Kerzaz souffre d'un climat aride et sévère, se caractérise par de faibles températures hivernales $\left(T \leq 6^{\circ} \mathrm{C}\right)$, des températures estivales élevées $\left(\mathrm{T} \geq 45^{\circ} \mathrm{C}\right)$, des vents de sables violents et une faible humidité de l'air malgré l'existante des palmeraies qui créent un microclimat. La pluviométrie est faible et irrégulière ( $<50 \mathrm{~mm} / \mathrm{an})$.

\subsection{La géomorphologie de l'oasis}

Kerzaz est caractérisée par la présence des alluvions. Ces alluvions se trouvent dans les lits d'oueds et les plaines inondables, ou dans leurs anciennes terrasses. Elles sont occupées par le Grand Erg occidental qui lui donne sa personnalité [3]. La géologie de Kerzaz dispose d'une sebkha «Sebkhat El Malah» qui renferme des couches de sels de table (NaCI) dont les potentialités sont énormes et à exploiter. Le Grand Erg Occidental est l'un des deux énormes mers de sable qui couvrent la majeur partie de l'Algérie, le Grand Erg Occidental traverse la plupart des oasis de la Saoura, parmi elles l'oasis de kerzaz entre Béchar et Adrar.

\subsubsection{Oued Saoura}

Oued Saoura a une longueur de $450 \mathrm{~km}$ et une largeur moyenne $100 \mathrm{~m}$ (figure 3) [4], c'est une convergence d'oued Zousfana et oued Guir à l'entrée de la commune d'Igli, les écoulements sont provoqués généralement par les précipitations sur le haut bassin, déferlent plus ou moins loin en aval, beaucoup atteignent une cuvette fermée: la sebkha El Mellah, par l'intermédiaire de l'oued Es Souireg qui descend vers le Nord-ouest à la sortie du Fourn el Kheneg. 


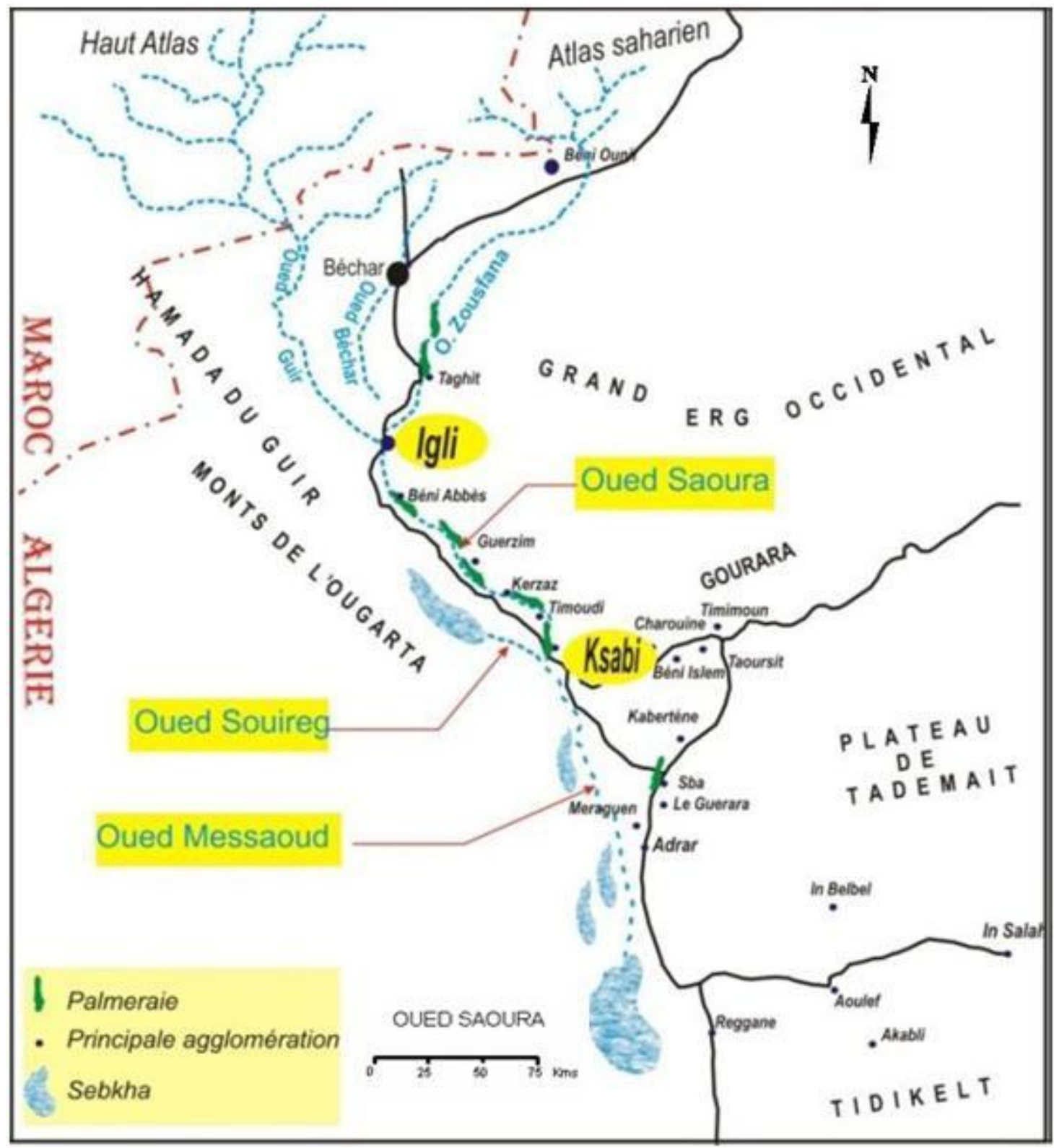

Fig.3. La carte du bassin versant d'oued Saoura [4]

Parmi les causes de raréfaction des crues d'oued Saoura est la réalisation du barrage Djorf Torba au niveau d'oued Guir [3]. Actuellement oued Saoura reçoit un volume de $6 \mathrm{Mm}^{3}$ d'eau (il est seulement la partie de contribution d'oued Zousfana), alors qu'il était de $206 \mathrm{Mm}^{3}$ avant la construction du barrage de Djorf Torba [5].

\subsubsection{Oued Megsem}

Il traverse le cœur de l'agglomération chef-lieu de Kerzaz, ce dernier représente un affluent d'oued Saoura. Il est formé de deux affluents (Chaabates): la Chaaba Sud-ouest et le Chaaba 
Sud-est. Cet oued représente un risque d'inondation en période des crues [6].

\subsubsection{La nappe du Grand Erg Occidental}

C'est une nappe phréatique qui représente la ressource souterraine la plus importante dans toute la Saoura. Les eaux de cette nappe alimentent les terrasses de la rive gauche d'oued Saoura où sont implantées des oasis.

\subsubsection{La nappe de l'oued}

Elle est alimentée par les crues d'oued Saoura. Même secs les oueds sahariens se distinguent par un courant souterrain d'inféro- flux.

\section{RESULTATS ET DISCUSSION}

\subsection{Le terrain agricole de l'oasis}

Même si l'oasis est pour les agronomes un lieu de production où s'organise et se concentre l'activité agricole, ses multiples autres fonctions évoluent dans le temps [7]. Selon le bulletin statistique de la wilaya de Béchar de l'année 2007, la commune de Kerzaz dispose d'une superficie agricole totale de 1.571 ha environ [2]. Les terrains non exploités et mis en valeur hors périmètre pour les nouveaux agriculteurs sont implantés d'une manière impropre (plus de 430 hectares), la plupart des implantations de ces terrains créent des obstacles dans les extensions avenir de l'oasis. la plupart de la surface agricole « jnans » de la palmeraie ne dépassent pas 0,5 hectare à cause de l'héritage et de l'exigüité de l'oasis [8], ces jnans sont ceinturés par des petits murets de terre avec une entrée de 1,5 à 2 mètre. L'ancienne palmeraie couvre à peu près de 222 hectares avec plus de 21250 palmiers existants.

Le nombre de personnes qui pratiquent l'agriculture est plus de 482 personnes, 234 sont des fellahs traditionnels et 13 personnes sont des éleveurs. La palmeraie de Kerzaz est caractérisée essentiellement par un étage supérieur de palmier dattier qui offre un microclimat pour les autres étages inférieurs: les arbres fruitiers et les cultures sous-jacentes [2].

Actuellement, plusieurs d'hectares de l'oasis sont abandonnés et deviennent morts en raison de diminution de la rentabilité de l'agriculture traditionnelle et de faible niveau des eaux de nappes.

\subsubsection{Le mode d'irrigation de Kerzaz}


A Kerzaz en se trouve plus de 140 puits à chadoufs traditionnels appelés puits de khottara (figure 4), l'irrigant utilise une longue perche dont une extrémité porte un contrepoids qui fait remonter sans trop d'efforts la poche de cuir (tagnint, dalou) remplie d'eau suspendue à l'autre extrémité. En peu se trouvent des puits collectifs entre les frères et des cousins proches de la grande famille. Aujourd'hui la majorité de ses puits sont équipés par des motopompes.
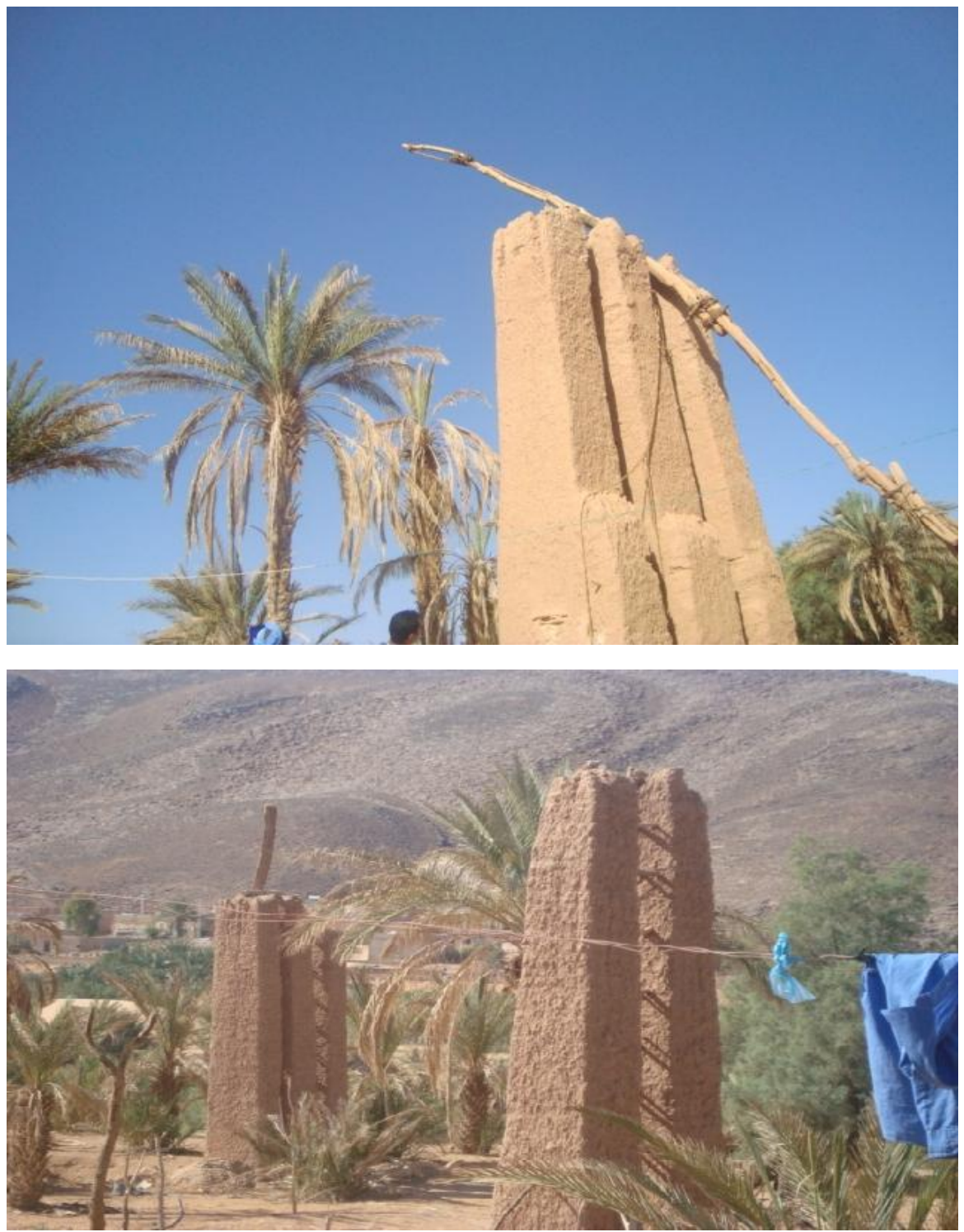

Fig.4. Puits de Khottara- l'oasis de Kerzaz. 2013

La profondeur des puits varie entre 4 à 12 mètres pour les puits traditionnels. Les puits les plus profonds sont rencontrés pris de Ksar de Kerzaz (tableau 1). 
Tableau 1. Inventaire des puits et forages dans l'oasis de Kerzaz. (Source: direction des services agricoles de Bechar), 2013:

\begin{tabular}{ccc}
\hline \multirow{2}{*}{ FORAGES } & Nombre & 14 \\
& Débit $(1 / \mathrm{s})$ & 26 \\
& Production $\left(\mathbf{M}^{3} / \mathbf{J}\right)$ & 2199.60 \\
\hline \multirow{2}{*}{ PUITS } & Nombre & 74 \\
& Débit $(1 / \mathrm{s})$ & 17.13 \\
& Production $\left(\mathbf{M}^{3} / \mathbf{J}\right)$ & 1450.00 \\
& Total production $\left(\mathbf{M}^{\mathbf{3}} / \mathbf{J}\right)$ & $3.649,60$ \\
\hline
\end{tabular}

Les agriculteurs au sein de l'oasis dépendent pour arroser ses terres sur un ensemble de réseaux d'irrigation non bétonnées de forme hiérarchisée de seguias (figure 5), ces canaux sont terminés toujours par un bassin de rétention «majen » en béton armé, les majens ont des dimensions différentes, les fellahs distribuent l'eau à l'intérieur de la parcelle par gravité. Les seguias sont aujourd'hui Souffrent de la destruction du fait des crues violentes comme la crue d'octobre 2008 et de négligence. Les fellahs substituent les systèmes traditionnels d'exhaure par les motopompes. Ils sont passés de la manipulation de chadouf vers la mécanisation de l'exhaure par des pompes de grands débits de ces mêmes puits traditionnels.

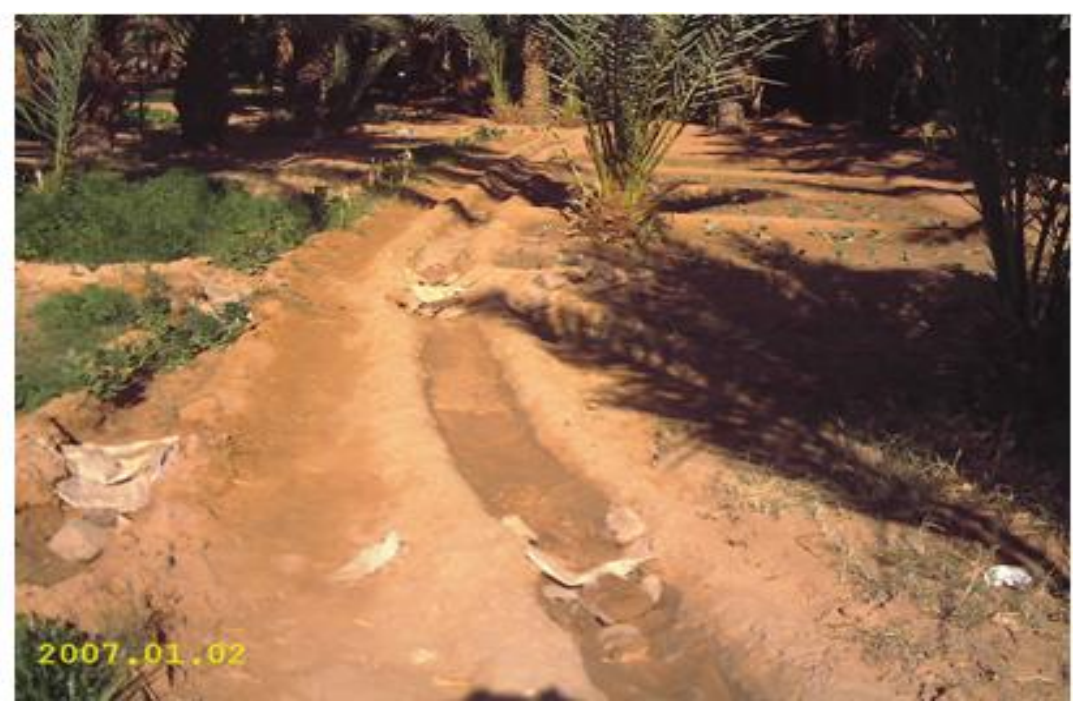

Fig.5. Canal d'irrigation (seguia) en sable à l'oasis de Kerzaz. 2007 


\subsubsection{Système moderne d'irrigation}

La multiplication anarchique des forages au sein de l'oasis équipés par des motopompes, conduit à l'épuisement des nappes souterraines. Le patrimoine environnemental de l'oasis déjà fragilisé par l'effet des périodes récurrentes de sécheresses et soumis à l'aridité du climat et à ces modes irrationnels d'exploitation (surexploitation, surpâturage, pompage excessif de l'eau, etc.) est aujourd'hui très dégradé. En plus, le barrage de Djorf Torba et l'utilisation du pompage ont aggravés l'état des nappes de l'oasis de Kerzaz [6]. Les forages ont générés un accroissement rapide des débits prélevés sur les ressources hydrauliques fossiles et très peu renouvelables du sous-sol saharien [9].

\subsection{Extensions agricoles à kerzaz}

Un espace agricole constitué par des terres agricoles créées dans le cadre de l'accession à la propriété foncière Agricole (A.P.F.A). Ce phénomène a pris de l'ampleur à partir de 1985/86 avec le début de l'application de la loi sur l'accession à la propriété foncière agricole en Algérie (APFA). Ces dernières se sont développées proches des oasis; sur des zones d'épandage des oueds ou sur toutes terres jugées par les fellahs aptes à être mis en valeur (cas des terres hors périmètre agricoles). À Kerzaz plus de 430 Ha étaient enregistrés par ce programme en 2007 (figure 6).

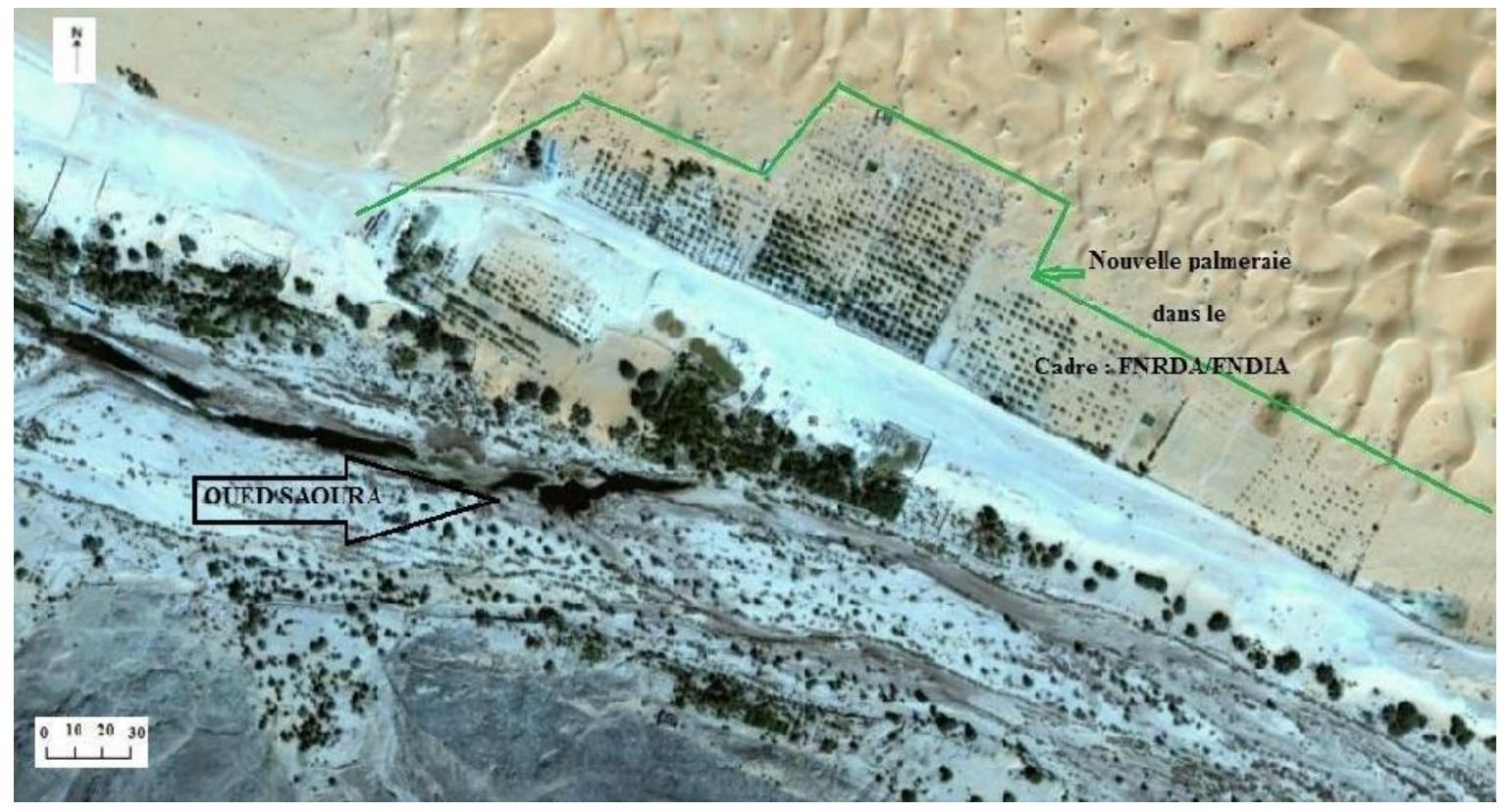

Fig.6. L'extension agricole de la palmeraie de Kerzaz [2]. 


\subsection{Phénomène de la salinité des nappes}

Le problème de salinité des eaux souterraines de l'oasis de Kerzaz est causé par plusieurs facteurs humains et naturels, engendrant des sérieux problèmes d'irrigation [10]. Les analyses hydro-chimiques montrent une évolution spatio-temporelle de l'alarmante de la salinité: l'assèchement des nappes de l'oasis dû d'une part à l'impact de la construction du barrage de Djorf Torba, l'exploitation des nappes par les motopompes avec la prolifération anarchique des puits et d'autre part à la forte évaporation qui règne, ils ont sérieusement affectés les eaux souterraines. Ces facteurs conduisent à une accélération spectaculaire de ce phénomène destructeur de tout le système oasien de cette région.

Pour étudier l'impact de l'exploitation des systèmes modernes sur l'évolution chimique de l'eau de la nappe qui alimente les puits de Kerzaz, on utilise les données indiquées dans le tableau 2, la première réalisée durant l'année 1966 avant l'inauguration du barrage Djorf Torba, la deuxième compagne d'analyse exécutée en 2005 et la dernière après la crue d'Octobre 2008. Les analyses des prélèvements des années 2005 et 2009 étaient faites par l'agence nationale des ressources en eaux (A.N.R.H de Béchar) [2]. Pour la période 1966, les résultats d'analyses sont extraits des anciens inventaires (figure 7) [2].

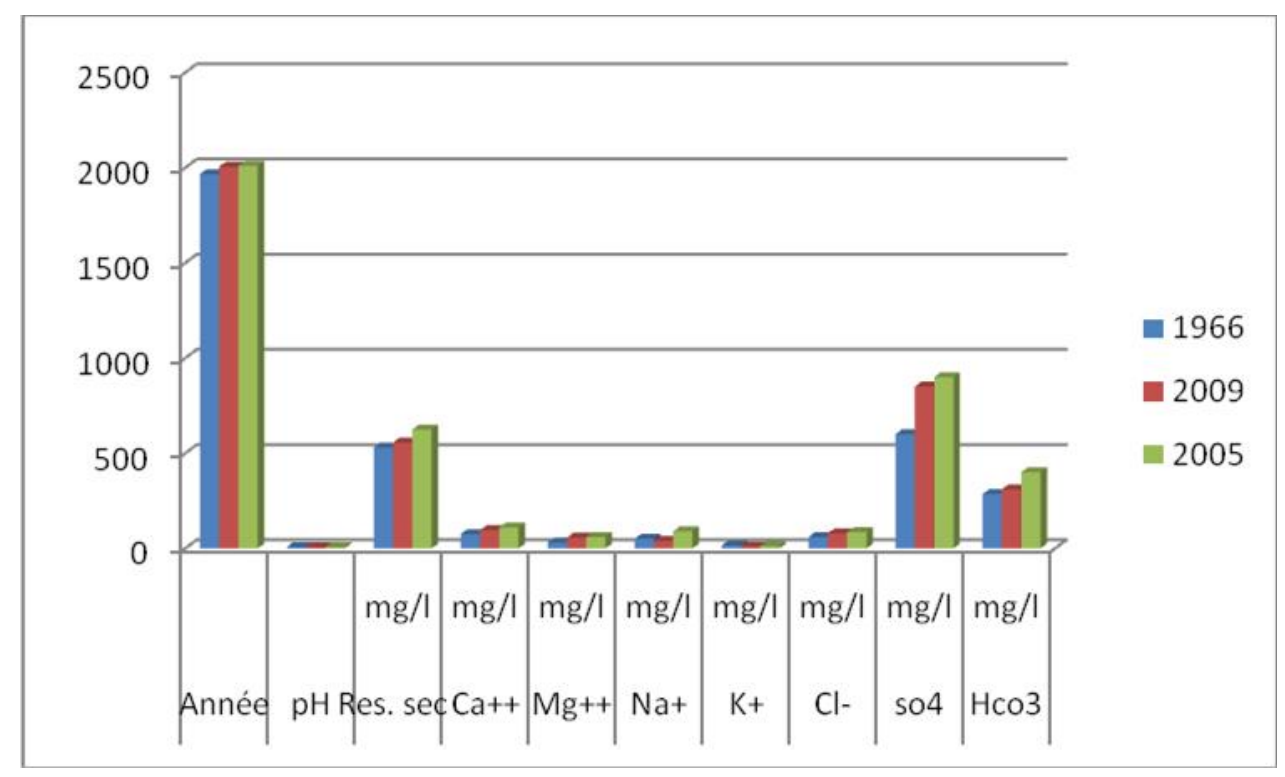

Fig.7. Evolution hydro-chimique des eaux de la nappe du grand erg occidental [2].

Pour les analyses de l'année 1966, les eaux souterraines ont des concentrations en sel 
relativement faibles en comparaison à celles de 2005 et 2009 (tableau 2). La diminution du gain de l'oued Saoura d'après la réalisation du barrage Djorf Torba au niveau d'oued Guir, quelle est la ressource la plus importante pour oued Saoura, l'évolution anarchique de l'utilisation de pompage mena à une mauvaise qualité des eaux souterraines. L'évolution de la salinité explique le nombre élevé des puits à chadouf abandonnées.

Tableau 2. Analyse hydro-chimique des eaux de la nappe du Grand Erg Occidental [2].

\begin{tabular}{lccccccccccc}
\hline Groupe d'eau & Année & $\mathbf{p H}$ & $\mathbf{R e s . ~ s e c}$ & $\mathbf{C a}^{++}$ & $\mathbf{M g}^{++}$ & $\mathbf{N a}^{+}$ & $\mathbf{K}^{+}$ & $\mathbf{C l}^{-}$ & $\mathbf{S O}_{4}$ & $\mathbf{H C O}_{3}$ \\
& & & $\mathbf{m g} / \mathbf{l}$ & $\mathbf{m g} / \mathbf{l}$ & $\mathbf{m g} / \mathbf{l}$ & $\mathbf{m g} / \mathbf{l}$ & $\mathbf{m g} / \mathbf{l}$ & $\mathbf{m g} / \mathbf{l}$ & $\mathbf{m g} / \mathbf{l}$ & $\mathbf{m g} / \mathbf{l}$ \\
Grand Erg & $\mathbf{1 9 6 6}$ & 7.6 & 530 & 75 & 30 & 50 & 15 & 60 & 600 & 285 \\
Occidental & & & & & & & & & & \\
Grand Erg & $\mathbf{2 0 0 5}$ & 7.7 & 556 & 96 & 57 & 40 & 10 & 80 & 850 & 310 \\
Occidental & & & & & & & & & & \\
Grand Erg & $\mathbf{2 0 0 9}$ & 7.5 & 625 & 110 & 60 & 90 & 12 & 85 & 900 & 400 \\
Occidental & & & & & & & & & & \\
\hline
\end{tabular}

\section{CONCLUSION}

Les savoirs hydrauliques traditionnels assuraient l'épanouissement des jardins de Kerzaz depuis des siècles mais aujourd'hui ces systèmes traditionnels souffrent de plusieurs crises telles que: L'envahissement des techniques modernes d'irrigation, la surexploitation des nappes phréatiques, l'incompatibilité des systèmes de partage avec des vastes zones d'arrosage. Les puits de Khottara sont aujourd'hui Souffrent de la destruction du fait des crues violentes d'oued Saoura et d'oued Megsem,

Il y a deux causes lesquelles provoquent le problème de la salinisation:

- Les puits équipés par des motopompes existants (plus de 140 puits de khotorra) prennent l'eau douce souterraine qui provient du Grand Erg. La surexploitation de la nappe accentue le phénomène de salinité et ne peut pas satisfaire la demande en eau d'irrigation et de consommation de l'agglomération.

- Les eaux de la nappe de l'oued sont normalement douces. elles sont devenues salées à cause du manque de lessivage après la réalisation du barrage Djorf Torba au niveau 
d'oued Guir qui alimente Oued Saoura (le flux d'eau normal et régulier de l'oued Saoura ne vient plus à cause du grand barrage «Djorf Torba » et il n'y a plus un lessivage du sel).

Pour sauvegarder et développer cette importante palmeraie du Sud-ouest algérien, il s'agit notamment de:

- L'adaptation des techniques de distribution et de partage pour se conformer avec la modernité.

- Empêcher le creusement des forages par la création d'un périmètre de protection des eaux souterraines autour de l'oasis.

- Le bétonnage des canaux (seguias) en terre pour réduire les pertes et les fuites d'eaux.

- Conscientiser les paysans de l'importance du patrimoine oasien et de protéger pour les générations futures.

Finalement, l'exploitation excessive des nappes souterraines par les techniques modernes d'irrigation et le non réalimentation d'oued Saoura par oued Guir explique la salinité du sol et le nombre élevé des puits de khottara abandonnés.

\section{BIBLIOGRAPHIE}

[1] Tarek M., Le partage de l'eau dans l'oasis de Figuig (Maroc oriental) Approche historique et archéologique. Mélanges de la Casa de Velázquez, Espagne, 2006, pp. 61-81

[2] Bouterra A., L'impact de l'utilisation des systèmes modernes d'irrigation sur les oasis de la Saoura: cas de Béni Abbes et Kerzaz, mémoire de Magister. Université TAHRI Mohamed Béchar, Algérie, 2014, 144 p.

[3] Merzougui T., Valorisation des ressources en eau de la haute vallée de la Saoura (entre Taghit et kerzaz) mémoire d'ingénieur, Univ Sci Tech d'Oran Algérie, 1998, 175 p.

[4] Ameri S., Mekkaoui A.; Merzougui T., La Saoura, espace hydraulique en crise : impacts des facteurs naturels et actions anthropiques sur les ressources hydriques (Sud-ouest, Algérie). International journal for environment \& global climate change, 2 (3), 2014, pp. $55-65$.

[5] Daoudi, Kadouri F., Etude comparative entre les technique de captage et de partage des 
eaux dans l'oasis de kerzaz au Sud-ouest algérien, mémoire d'ingénieur. université de Saida, Algérie, 2002, 135 p.

[6] Lefkir A., Elaboration de cartes thématiques sur les ressources hydriques dans une zone saharienne, cas de la willaya de Bechar, Seminaire national sur l'eau et l'environnement SN2E, Bechar, Algérie, 2005.

[7] Toutain G., Dolle V., Ferry M., Situation des systèmes oasiens en régions chaudes, Les systèmes agricoles oasiens. Montpellier: (Options Méditerranéennes: Série A. Sémin aires Méditerranéens; n. 11) CIHEAM, 1990, pp. 7-18.

[8] Benmoussa, Y., Rezzoug C., Remini B., Belhadj M., Ressources et partage de l'eau dans l'oasis de Kenadsa (Sud-Ouest Algerien): approche historique et constat, Cinq Continents, revue roumaine de géographie 5(11), 2015, pp. 81-92.

[9] Kassah A., Oasis et aménagement en zones arides. Enjeux, défis et stratégies. Gestion des ressources naturelles et développement durable des systèmes oasiens du Nefzaoua, Douz, Tunisie. Cirad (6), 2009.

[10] Merzougui T., kabour A.,bouanani A., mekkaoui A., chebbah L., Un modèle de gestion intégrée des ressources en eau dans une zone hyperaride : Cas de l'oasis de Béni Abbès (Vallée de la Saoura, Sud-ouest algérien), 13e Congrès Mondial de l'Eau, Montpellier, France, 2008.

\section{How to cite this article:}

C. Rezzoug, B. Remini and S. Hamoudi. The knowledges of traditional irrigation in the oasis of Kerzaz in southwest Algerian: legacy and development. J. Fundam. Appl. Sci., 2017, 9(1), 261-273. 